\title{
Laparoscopic roux en y gastric bypass: a good operation losing ground
}

\begin{abstract}
Abbreviations: RYGB, roux en y gastric bypass; VBG, vertical banded gastroplasty; LAGB, laparoscopic adjustable gastric banding; JI, jejunoileal; LRYGB, laparoscopic roux en y gastric bypass; LSG, laparoscopic sleeve gastrectomy
\end{abstract}

\section{Editorial}

Obesity is currently a pandemic of epic proportions. The ramifications and the consequences of morbid obesity will continue for generations. Bariatric surgery cannot be the only solution for morbid obesity. However, it is currently the only effective proven long term treatment available for morbid obesity.

Bariatric surgery history have witnessed many operations go by the way side because of ineffectiveness or major complications. Roux en Y gastric bypass (RYGB) has stood the test of time and has been the golden standard for surgical therapy in bariatric surgery for over 40years. Two examples of operations that have declined in use because of lack of effectiveness are the Vertical Banded Gastroplasty (VBG) and the Laparoscopic Adjustable Gastric Banding (LAGB). An example of an operation that disappeared due to complications is the jejunoileal (JI) bypass.

RYGB is the only commonly performed operation with more than $20 y e a r$ of outcomes reported showing durability. The possibility of performing RYGB laparoscopically has reduced the peri-operative complications even further at the expense of a steeper learning curve. Nevertheless, for more than a decade laparoscopic RYGB has been the most common operation performed worldwide. However, LRYGB has a steep learning curve, involves re routing the small intestine, has potential long term complications and very little options if patients regain weight. Despite the effectiveness of LRYGB, its superior outcomes and durability, more patients and surgeons are preferring to undergo and perform laparoscopic sleeve gastrectomy (LSG). LSG was an operation originally performed as the first stage of the duodenal switch to decrease the peri-operative morbidity and mortality in morbidly super obese patients. However, many super obese patients did not require the second stage and the LSG is now performed as a primary operation for morbid obesity. In a recent report of the world wide trend of bariatric surgery and for the first time in more than 40years LRYGB will soon not become the most commonly performed operation and LSG has overtaken the LRYGB not only in Asia and Europe but even in the USA.

LRYGB is not becoming less favorable because of lack of effectiveness or high complications like the VBG, LAGB and the JI bypass. Nevertheless, LRYGB has been over taken by the LSG, an operation that has no data past 10years in the published literature. However, LSG has been shown to have lower peri-operative complications than LRYGB, less weight loss and less resolution of co morbid conditions. The steep learning curve, re routing of the small bowel, potential known long term complications and the paucity of
Volume 2 Issue I - 2015

\begin{abstract}
Abdelrahman Nimeri
Bariatric \& Metabolic Institute (BMI), Sheikh Khalifa Medical City, United Arab Emirates

Correspondence: Abdelrahman Nimeri, Chief, Division of General, Thoracic \& Vascular Surgery! Director, Bariatric \& Metabolic Institute (BMI), Sheikh Khalifa Medical City, United Arab Emirates, Tel+97I50208086I, Email nimeri@gmail.com

Received: December 22, 2014 | Published: January 06, 2015
\end{abstract}

options to treat patients with weight recidivism after LRYGB are not the main reasons for the decrease in LRYGB numbers. In contrast, the short learning curve, no re routing of the small bowel, unknown long term complications and the many options to revise a LSG to a duodenal switch or LRYGB are the reasons patients and surgeons are choosing the LSG. The question remains whether we are only witnessing a very effective restrictive operation like the VBG without long term outcomes. ${ }^{1-6}$

\section{Acknowledgements}

None.

\section{Conflict of interest}

The author declares there is no conflict of interest.

\section{References}

1. Sjostrom L, Lindroos AK, Peltonen M, et al. Lifestyle, diabetes, and cardiovascular risk factors 10 years after bariatric surgery. $N$ Engl $J$ Med. 2004;351(26):2683-2693.

2. Sjostrom L, Narbro K, Sjostromet D, et al. Effects of bariatric surgery on mortality in Swedish obese subjects. N Engl J Med. 2007;357(8):741752.

3. Van Rutte PW, Smulders JF, de Zoete JP, et al. Outcomes of sleeve gastrectomy as a primary bariatric procedure. Br J Surg. 2014;101(6):661668.

4. Buchwald H, Oien DM. Metabolic/bariatric surgery worldwide 2011. Obes Surg. 2013;23(4):427-436.

5. Higa $\mathrm{K}$, Boone $\mathrm{K}$, Ho T, et al. Laparoscopic Roux-en-Y gastric bypass for morbid obesity: technique and preliminary results of our first 400 patients. Arch Surg. 2000;135(9):1029-1034.

6. Hutter MM, Schirmer BD, Jones DB, et al. First report from the American College of Surgeons Bariatric Surgery Center Network: laparoscopic sleeve gastrectomy has morbidity and effectiveness positioned between the band and the bypass. Ann Surg. 2011;254(3):410-422. 